\title{
Agenda for the 33rd General Assembly of the European Association for the Study of Diabetes to be held in Helsinki, in Hall C1 of the Helsinki Fair Center, 22nd July 1997 from 12.15-13.30 hours.
}

1. Minutes of the 32nd General Assembly, Vienna 1996

2. Reports
a) President
b) Honorary Treasurer
Dr. M. Berger
c) Honorary Auditors
Dr. R. J. Heine
Dr. P.J. Guillausseau
d) Honorary Secretary
Dr. D. Pipelecrs
c) Editor in Chief, Diabetologia
Dr. U.J. Eriksson
Dr. E. Ferrannini

3. Elections

a) President 1998-2001 in place of

Dr. M. Berger (D)

retiring by rotation

b) Editor-in-Chief, Diabetologia

in place of

1998-2001

Dr. E. Ferrannini (I)

retiring by rotation

c) Adjunct Honorary Secretary

adjunct to

1998-1999

Dr. U.J. Eriksson (S)

d) Council Members

in place of

1998-2001

Dr. M. Benroubi (GR)

Dr. R.W. Bilous (UK)

Dr. H. Yki-Järvinen (FIN)

4. Postgraduate Education

Dr. AJM Boulton (UK)

Sub-Committec

5. Study Groups

6. Honorary Membership Dr. E. Shafrir

7. Any other business 